\title{
Spontaneous intramyometrial pregnancy mimicking an intramural myoma: a diagnostic challenge
}

\author{
Maria-Laura Marotta $\cdot$ Jacques Donnez • \\ Nathalie Michaux • Michaël Dupont • Monique Delos • \\ Olivier Donnez
}

Received: 30 March 2012 / Accepted: 11 April 2012 / Published online: 17 June 2012

(C) Springer-Verlag 2012

\begin{abstract}
This study describes the diagnosis and treatment of a spontaneous intramyometrial pregnancy by a case report and review of the literature at a university hospital center. A 24-year-old woman presented with a spontaneous intramyometrial pregnancy without any previous uterine surgery. Successful laparoscopic resection and myometrial correction were performed. The presence of trophoblastic tissue was confirmed and the myometrium revealed complete healing at postoperative MRI. Intramyometrial pregnancy can occur without previous uterine surgery or uterine manipulation and may be difficult to diagnose. Conservative laparoscopic excision is possible without damage to myometrial integrity.
\end{abstract}

M.-L. Marotta $(\triangle) \cdot$ N. Michaux $\cdot$ O. Donnez

Department of Gynecology, CHU Mont-Godinne,

Université Catholique de Louvain,

Av. G. Thérasse, 1,

5530 Yvoir, Belgium

e-mail: maria-laura.marotta@uclouvain.be

J. Donnez

Pôle de Recherche en Gynécologie,

Institut de Recherche Expérimentale et Clinique (IREC),

Université Catholique de Louvain (UCL),

1200 Brussels, Belgium

J. Donnez

Department of Gynecology, Cliniques Universitaires Saint-Luc,

1200 Brussels, Belgium

\section{Dupont}

Department of Radiology, CHU Mont-Godinne,

Université Catholique de Louvain,

5530 Yvoir, Belgium

\section{Delos}

Department of Anatomopathology, CHU Mont-Godinne,

Université Catholique de Louvain,

5530 Yvoir, Belgium
Keywords Ectopic pregnancy · Intramyometrial · Myometrial pregnancy

\section{Introduction}

Intramyometrial pregnancy is a very rare type of ectopic pregnancy. This uncommon condition has been estimated to occur in less than $1 \%$ of all ectopic gestations [1]. An intramyometrial pregnancy is located within the uterine wall, completely surrounded by myometrium, and separated from the uterine cavity and fallopian tubes [1], but confusion remains in the literature as to its physiopathology. Ong et al. explain intramyometrial ectopic pregnancy by increased lytic activity of the syncytiotrophoblast and defective decidualization, allowing the conceptus to penetrate the myometrium [1]. But, as described by Pijnenborg et al., in normal pregnancy, the decidua attracts the trophoblast and both intravascular and interstitial trophoblasts invade the inner myometrium [2]. Risk factors have been identified to explain the pathogenesis, including previous uterine trauma causing a sinus tract within the endometrium $[1,3,4]$, implantation on a focus of intramural adenomyosis $[5,6]$, or difficult embryo transfer with a false passage [1, 7-9]. Others have described invasion of the uterine wall by placenta accreta and subsequent growth of the fetus deep within the myometrium [10].

Due to late and often difficult ultrasonographic diagnosis, the gestational sac can grow deep in the myometrium and cause the main complication, namely uterine rupture associated with life-threatening hemorrhage reported to occur between week 11 and week 30 of gestation [1]. To our knowledge, this is the first reported case of intramyometrial pregnancy to occur without any previous surgery or uterine manipulation. 


\section{Case report}

A 24-year-old woman with a history of spontaneous abortion without dilatation-curettage was referred as an emergency case to our department with acute pelvic pain and suspected ectopic pregnancy. Vaginal examination provoked acute pain upon palpation of the right adnexa, while the uterus appeared to be of normal size. HCG was $102 \mathrm{IU} / 1$, and vaginal ultrasound confirmed a normal-sized uterus, but containing a heterogeneous subserosal mass measuring $35 \times$ $30 \mathrm{~mm}$ and regularly circumscribed. An adenomyoma or necrotic uterine myoma was suspected. Both adnexa looked normal, but the Douglas pouch revealed the presence of blood. Because of acute pain at vaginal examination, low HCG level, and ultrasound status, ectopic pregnancy was suspected and diagnostic laparoscopy was performed.

At laparoscopy, the uterus showed a subserosal $3 \mathrm{~cm}$ mass located on the right side of the uterus below the right utero-ovarian ligament. Fifty milliliters of blood was found in the Douglas pouch. The fallopian tubes were both unremarkable, and no ectopic pregnancy was identified. The abdominal cavity was flushed with physiological serum. On day 1, the HCG level was $44 \mathrm{IU} / 1$ and the patient did not present with any abdominal pain. She was then discharged from the hospital, and monitoring of HCG levels was proposed. On day 10, her HCG was less than $1.2 \mathrm{IU} / \mathrm{ml}$. One week after the first laparoscopy, vaginal examination revealed a 7-week-sized uterus, with tenderness on the right side.

Pelvic MRI was performed and confirmed the presence of a right lateral intramyometrial mass. Coronal and axial views of T2-weighted MRI images revealed a $35 \mathrm{~mm}$ heterogeneous mass at some distance from the uterine cavity (Fig. 1a, b). T1-weighted images (Fig. 1c, d) showed a discretely hyperintense mass. The right uterine horn was not distorted by the mass. The rest of the myometrium looked normal, as did both adnexa, and no uterine adenomyosis was found. A differential diagnosis was made between an intramural myoma in necrobiosis and intramyometrial pregnancy, but the T2-weighted signal was too heterogeneous.

Laparoscopic excision of the lesion was then proposed. Laparoscopic view of the right side of the uterus (Fig. 2a) confirmed a subserosal deformation of the myometrium below the right utero-ovarian ligament far from the right tube and uterine horn. To avoid bleeding after opening the myometrium, the right uterine artery was clipped at its origin (Fig. 2b). The uterine serosa and superficial layers of the myometrium were then opened using $\mathrm{CO}_{2}$ laser (power $50 \mathrm{~W}$ ) (Lumenis-Sharplan, Yokneam, Israel) at a safe distance from the right fallopian tube and right utero-ovarian ligament. The observed myometrial deformation corresponded to an intramyometrial cavity full of blood clots and fibrotic tissue (Fig. 2c). Intraoperative hysteroscopy was then performed and
Fig. 1 a Axial T2-weighted image revealed a wellcircumscribed heterogeneous mass (white arrow) subserously located far from the myometrial cavity. b Coronal view of a T2weighted image showed the subserosal mass (white arrow) without any deformation of the right uterine horn. c Axial T1weighted image with saturation of fatty tissue and injection of gadolinium into the slightly hypointense right uterine mass (white arrow), with hypocaptation of gadolinium. d Coronal T1-weighted image of the uterine mass (white arrow)
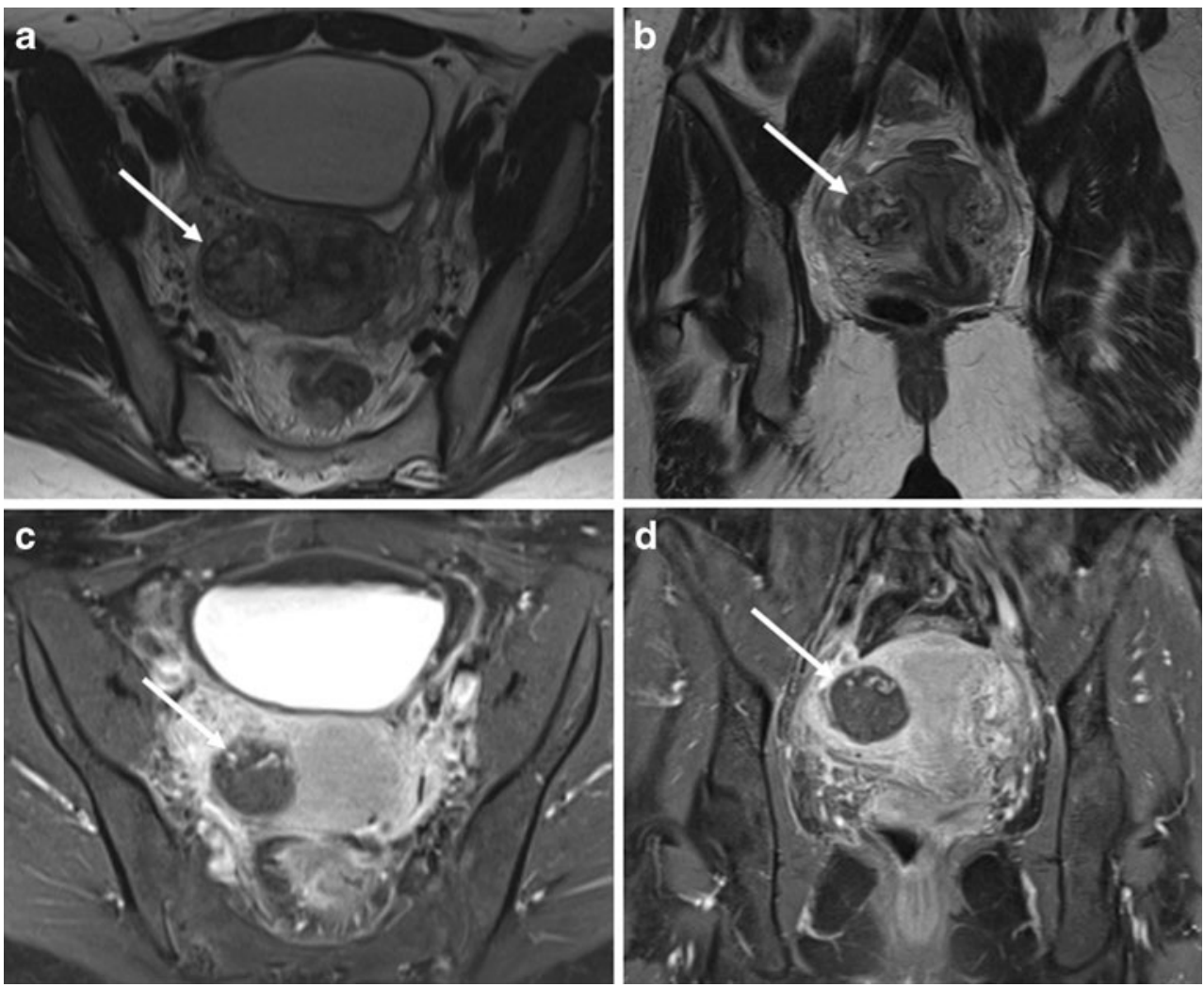
Fig. 2 a Laparoscopic view of the right side of the uterus with the subserosal mass located below the insertion of the right utero-ovarian ligament and tube (white and black arrows, respectively). b Laparoscopic view of the right uterine artery clipped at its origin (white arrow) to avoid intraoperative bleeding. c Laparoscopic view of the opened subserosal mass, revealing an intramyometrial cavity full of blood clots and fibrotic tissue (black arrow). d Final view of the two-layer running suture (white arrow)
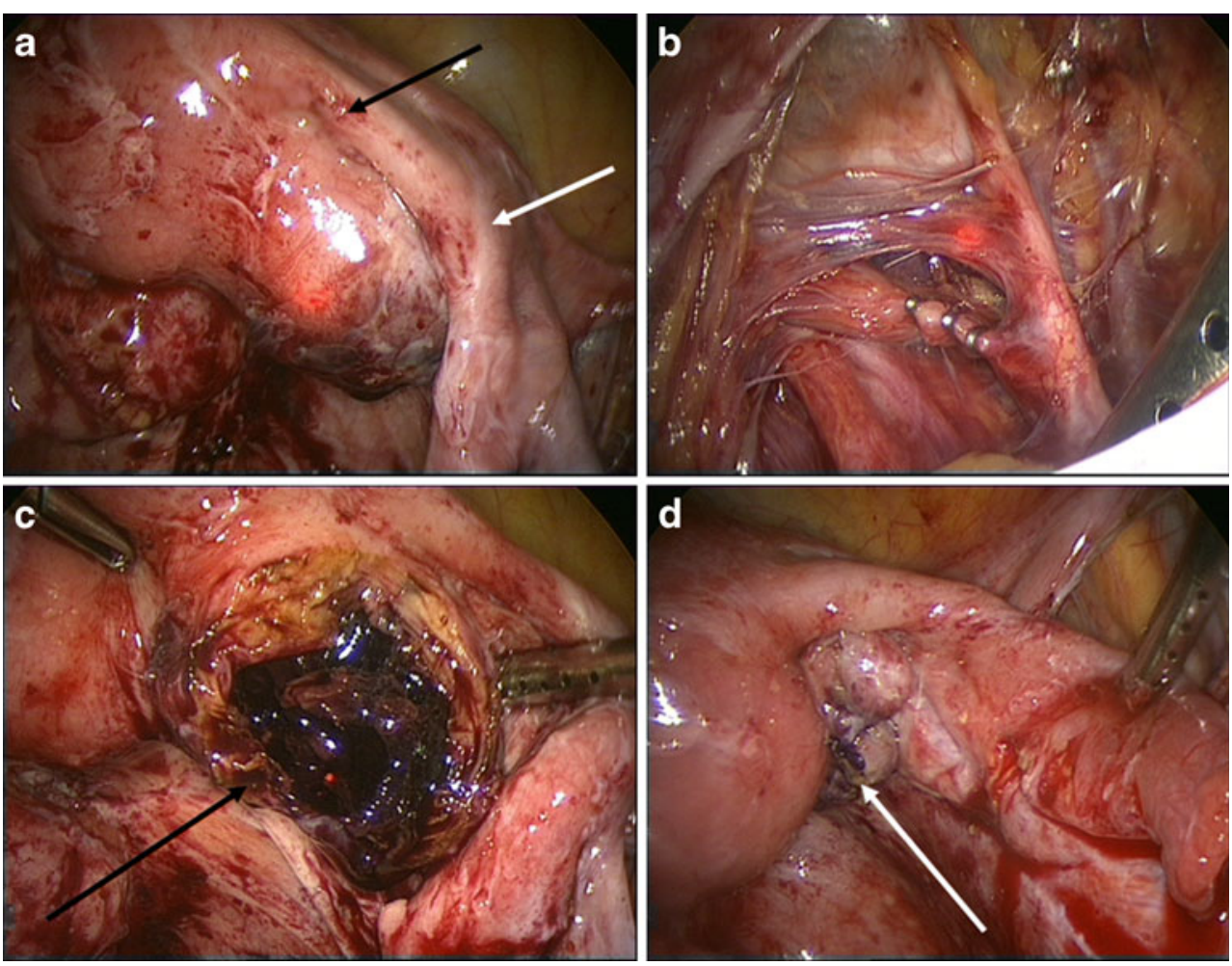

revealed a normal uterine cavity and normal ostia. The endometrium covering the right side of the uterine cavity also looked unremarkable. There were no visible fistulas between the uterine cavity and intramyometrial cavity. After removing all the fibrotic tissue and washing the myometrial cavity, the myometrium was closed in two layers using a running suture of Quill $^{\mathrm{TM}}$ SRS 0 (Angiotech, Reading, USA). The final result can be seen in Fig. 2d. In order to avoid more myometrial injury, no biopsy of the surrounding myometrium was performed. No drain was left in the peritoneal cavity, and the patient was discharged from hospital on day 1. Histologic examination of the excised tissue revealed degenerated villous tissue, proving the presence of a pregnancy inside the myometrium (Fig. 3a).
Pelvic MRI performed 3 months after surgery showed complete healing of the myometrium. No uterine scarring was visible (Fig. 3b). Clinical examination revealed a normal-sized uterus, and the patient did not experience any abdominal pain.

\section{Discussion}

Intramyometrial ectopic pregnancy is uncommon. Its etiology remains unclear, but most authors attribute it to a sinus tract within the endometrium caused by previous uterine surgery or manipulation [1, 3, 4], adenomyosis [5, 6], or a difficult embryo transfer [1, 7-9]. Leyder et al. described intramyometrial
Fig. 3 a Histology (HES, $\times 10)$ confirmed the presence of blood clots with necrotic placental villi (black arrow). b T2-weighted MRI image performed 3 months after laparoscopy, showing complete healing of the right myometrial wall
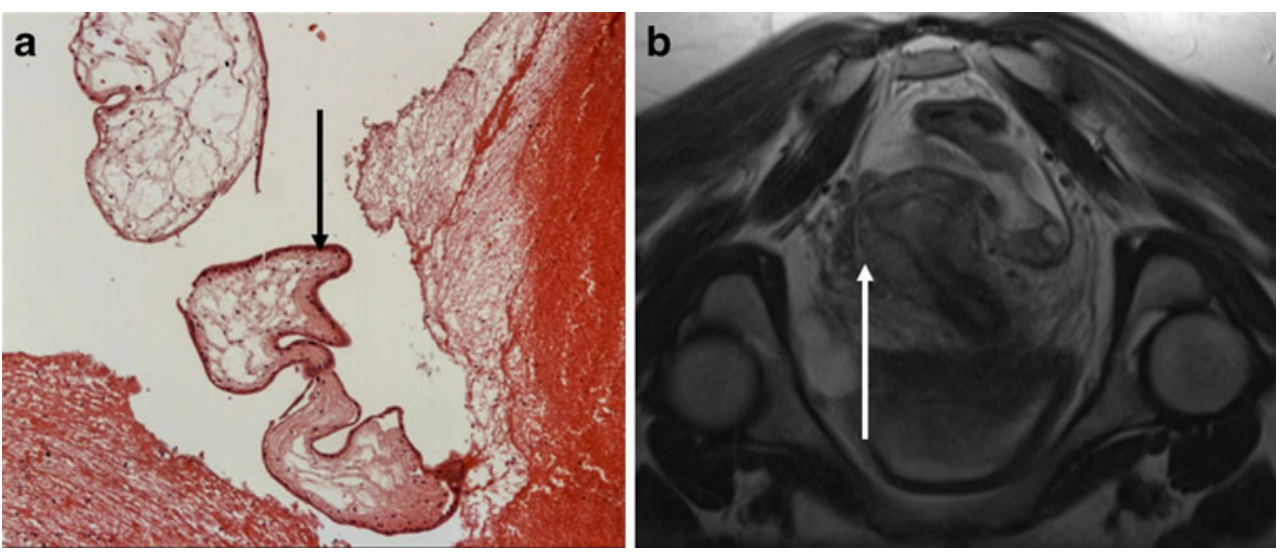


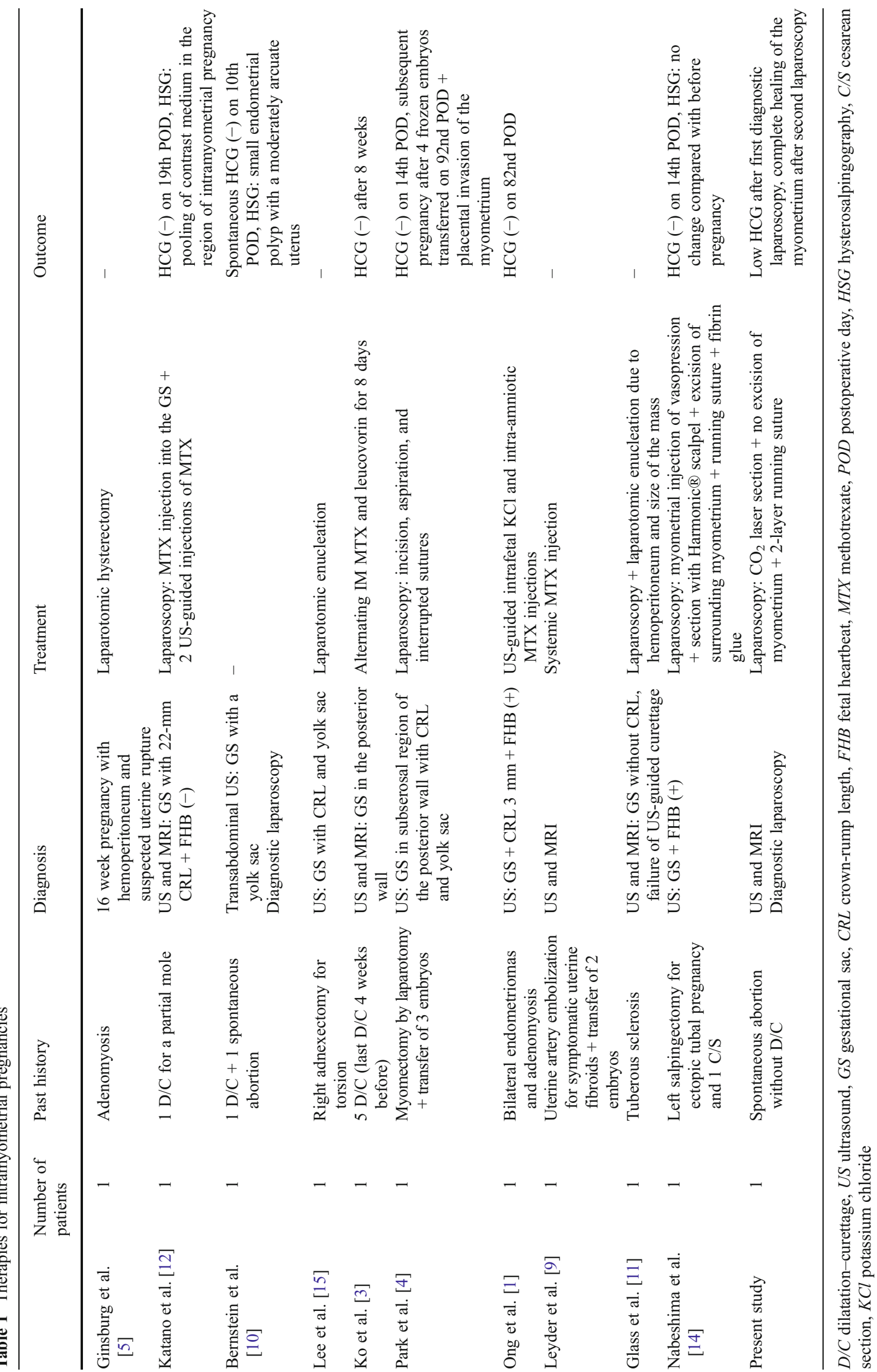


pregnancy after an embryo transfer following uterine artery embolization [9], and Glass et al. described a case in a woman with tuberous sclerosis [11]. In our case, there was no previous uterine surgery or previous intrauterine manipulation and no sinus tract was found during hysteroscopy. This supports the theory of uterine wall invasion by placenta accreta and subsequent growth of the fetus deep in the myometrium, as described by Bernstein et al. [10].

Differential diagnosis based on 2D ultrasound remains difficult and may suggest a number of possibilities, including interstitial or cornual ectopic pregnancy, degenerating myoma, pregnancy in a congenitally abnormal uterus [1], sarcoma or molar pregnancy [11], or unruptured intramyometrial pregnancy.

The use of pelvic MRI allows visualization of multiple scan planes and helps to define the distance between the intramyometrial pregnancy and the uterine cavity $[1,3,11$, 12]. Ko et al. proposed MRI to distinguish an unruptured intramyometrial pregnancy, which looks like a homogeneous hyperintense mass on T2-weighted images, from a ruptured one, that shows a heterogeneous signal intensity on T2-weighted images. On the other hand, contrast-enhanced T1-weighted images show tree-like, solid components resulting from hemorrhages at various times [3]. In our case, T2-weighted views revealed a heterogeneous mass, but T1weighted views showed a more homogeneous mass with only few remaining spots of contrast enhancement.

Treatment proposed in the literature differs and depends on the need to preserve future fertility. With enhancement by ultrasound and MRI, conservative medical treatment and conservative surgical treatment are options. All described therapies for intramyometrial pregnancies in the literature are summarized in Table 1.

Conservative medical management can be achieved using systemic intramuscular administration of methotrexate [13] or ultrasound-guided methotrexate injection into the chorionic cavity of patients with an intramyometrial pregnancy without a fetal heartbeat [12, 13]. Intra-amniotic injection of methotrexate can also be associated with intrafetal injection of potassium chloride in case of intramyometrial pregnancy with the presence of a fetal heartbeat [1].

Laparoscopic treatment with opening of the surrounding myometrium and suture by interrupted stitches was performed in a patient with an intramyometrial pregnancy in a myomectomy scar [4] and another who had undergone previous left salpingectomy [14]. Enucleation by laparotomy was also carried out for intramyometrial pregnancy after right adnexectomy [15] and due to massive hemoperitoneum [11].

Ginsburg et al. reported a case of suspected uterine rupture at 16 gestational weeks with hemoperitoneum. Hysterectomy was performed, and pathological examination showed the presence of an intramyometrial pregnancy [5].
In our case, despite a low HCG level, the patient still felt pain at vaginal examination. This led us to perform pelvic MRI, which was unable to differentiate between adenomyoma, necrotic leiomyoma, and intramyometrial pregnancy.

The low HCG level did not exclude uterine rupture. It can be compared to some ectopic pregnancies that occur with negative HCG levels. Ectopic pregnancy is due to growth of trophoblasts into the tubal mucosa that can cause erosion of tubal vessels, resulting in hematoma formation and tubal swelling. The persistence of ectopic pregnancy without rupture may lead, in some cases, to degeneration of trophoblasts and cessation of HCG production. Spontaneous abortion or repeated minor bleeding episodes in this type of chronic ectopic pregnancy can cause subsequent rupture associated with hemoperitoneum, with low or negative HCG levels [16]. Uterine conservative surgery was thus mandatory in our patient, with successful complete healing of the intramyometrial pregnancy site.

\section{Conclusion}

Intramyometrial ectopic pregnancy is a rare life-threatening entity. Our case suggests that spontaneous intramyometrial pregnancy can occur without any previous uterine surgery or intrauterine manipulation. Because of the paucity of cases described in the literature, diagnosis remains difficult and treatment tends to be conservative. Our case suggests that even in case of negative HCG levels, we cannot exclude ruptured intramyometrial pregnancy, and laparoscopic excision can be performed without damaging uterine wall integrity.

\section{References}

1. Ong C, Su LL, Chia D, Choolani M, Biswas A (2010) Sonographic diagnosis and successful medical management of an intramural ectopic pregnancy. J Clin Ultrasound 38(6):320-324

2. Pijnenborg R, Vercruysse L, Brosens I (2011) Deep placentation. Best Pract Res Clin Obstet Gynaecol 25(3):273-285

3. Ko HS, Lee Y, Lee HJ, Park IY, Chung DY, Kim SP, Park TC, Shin JC (2006) Sonographic and MR findings in 2 cases of intramural pregnancy treated conservatively. J Clin Ultrasound 34:356-360

4. Park WI, Jeon YM, Lee JY, Shin SY (2006) Subserosal pregnancy in a previous myomectomy site: a variant of intramural pregnancy. J Min Inv Gynecol 13:242-244

5. Ginsburg KA, Quereshi F, Thomas M, Snowman B (1989) Intramural ectopic pregnancy implanting in adenomyosis. Fertil Steril 51:354-356

6. Karakök M, Balat O, Sari I, Kocer NE, Erdogan R (2002) Early diagnosed intramural ectopic pregnancy associated with adenomyosis: report of an unusual case. Clin Exp Obstet Gynecol 29 (3):217-218 
7. Hamilton CJ, Legarth J, Jaroudi KA (1992) Intramural pregnancy after in vitro fertilization and embryo transfer. Fertil Steril 57:215217

8. Khalifa Y, Redgement CJ, Yazdani N, Taranissi M, Craft IL (1994) Intramural pregnancy following difficult embryo transfer. Hum Reprod 9:2427-2428

9. Leyder M, De Vos M, Popovic-Todorovic B, Dujardin M, Devroey P, Fatemi HM (2010) Intramyometrial ectopic pregnancy in an ICSI patient following uterine artery embolization. Reprod Biomed Online 20(6):831-835

10. Bernstein HB, Thrall MM, Clark WB (2001) Expectant management of intramural ectopic pregnancy. Obstet Gynecol 97:826-827

11. Glass T, Smith P, Hodges R, Holmes HJ (2010) Intramural pregnancy presenting in a patient with tuberous sclerosis. J Clin Ultrasound 38(7):393-396
12. Katano K, Ikuta K, Matsubara H, Oya N, Nishio M, Suzumori K (1999) A case of successful conservative chemotherapy for intramural pregnancy. Fertil Steril 72(4):744-746

13. Haimov-Kochman R, Sciaky-Tamir Y, Yanai N, Yagel S (2002) Conservative management of two ectopic pregnancies implanted in previous uterine scars. Ultrasound Obstet Gynecol 19(6):616-619

14. Nabeshima $H$, Nishimoto $M$, Utsunomiya $H$, Arai $M$, Ugajin $T$, Terada Y, Yaegashi N (2010) Total laparoscopic conservative surgery for an intramural ectopic pregnancy. Diagn Ther Endosc 2010:504062. Epub 2010 Oct 11

15. Lee GS, Hur SY, Kown I, Shin JC, Kim SP, Kim SJ (2005) Diagnosis of early intramural ectopic pregnancy. J Clin Ultrasound 33(4):190-192

16. Brennan DF, Kwatra S, Kelly M, Dunn M (2000) Chronic ectopic pregnancy - two cases of acute rupture despite negative beta HCG. J Emerg Med 19(3):249-254 